\title{
BLOWUP IN A PARTIAL DIFFERENTIAL EQUATION WITH CONSERVED FIRST INTEGRAL*
}

\author{
CHRIS BUDD $\dagger$, BILL DOLD $\dagger$, AND ANDREW STUART $\ddagger$
}

\begin{abstract}
A reaction-diffusion equation with a nonlocal term is studied. The nonlocal term acts to conserve the spatial integral of the unknown function as time evolves. Such equations give insight into biological and chemical problems where conservation properties predominate. The aim of the paper is to understand how the conservation property affects the nature of blowup.

The equation studied has a trivial steady solution that is proved to be stable. Existence of nontrivial steady solutions is proved, and their instability established numerically. Blowup is proved for sufficiently large initial data by using a comparison principle in Fourier space. The nature of the blowup is investigated by a combination of asymptotic and numerical calculations.
\end{abstract}

Key words. blowup, nonlocal source term, conserved integral, adaptive remeshing

AMS(MOS) subject classifications. 35B30, 35B35, 35B40, 35K57, 65N50

1. Introduction. It is well known that, for certain initial and boundary data, solutions of nonlinear parabolic equations of the form

$$
u_{t}=u_{x x}+f(u)
$$

may blow up in a finite time. Examples of such behaviour when the function $u(x, t)$ satisfies Dirichlet boundary conditions are given in [3], [8], [13], [17], and [18], and examples with Neumann boundary conditions are studied in [5].

In many evolutionary problems, the function $u(x, t)$ must satisfy additional constraints, and an example of such is that the integral of $u(x, t)$ is conserved throughout its evolution. This condition occurs naturally in problems arising in chemistry and mathematical biology in which the total mass of a chemical or an organism is conserved. Examples of such systems are the chemotaxis equations discussed in [20], [6], and [7]. Similar constraints occur for the Cahn-Hilliard equations of the phase density for a binary alloy [10], and other examples of nonlocal problems are given in [12] and [19].

In this paper, we consider the question of whether the addition of a constraint on the first integral of $u(x, t)$ affects the nature of its blowup behaviour. In a subsequent paper, we also consider the effect of including convective behaviour in (1.1), as well as the constraint on the first integral, to obtain a system of equations resulting from a similarity reduction of the Navier-Stokes equations.

Here we study the following problem defined on the interval $[0,1]$ :

$$
u_{t}=u_{x x}+u^{2}-K^{2}(t),
$$

together with the Neumann boundary conditions

$$
u_{x}(0)=u_{x}(1)=0
$$

and the integral constraint

$$
\int_{0}^{1} u(x, t) d x=0
$$

* Received by the editors September 25, 1991; accepted for publication (in revised form) April 23, 1992.

$\dagger$ School of Mathematics, University of Bristol, Bristol BS8 1TW, United Kingdom.

$\$$ School of Mathematical Sciences, University of Bath, Bath BA2 7AY, United Kingdom. The work of this author was supported by a Science and Engineering Research Council fellowship. 
We assume that for $t=0$

$$
u(x, 0)=u_{0}(x),
$$

where $u_{0}(x)$ satisfies the integral constraint.

The solutions we consider are those that are sufficiently smooth to lie in the spaces

$$
u(x, t) \in H^{1}(\Omega) \times C^{1}(0, T), \quad K^{2} \in C^{1}(0, T) .
$$

This problem has two boundary conditions and an integral constraint. To compensate for this, the function $K(t)$ is an unknown and must be determined as part of the solution. Indeed, a simple calculation shows that

$$
K^{2}(t)=\int_{0}^{1} u^{2}(x, t) d x
$$

Following [5], we may apply the theory of semigroups [15] to deduce that, for sufficiently smooth initial data $u_{0}(x)$, problem (1.2)-(1.4) has a solution $u(x, t)$ local in time [9]. For the remainder of this paper, we always assume that $u_{0}(x)$ is smooth enough for this to occur, and we study the resulting evolution of the solution. In particular, we examine solutions that blow up (both in the $L_{2}$ and $L_{\infty}$ norms) at some finite time $T$.

Problems of the form (1.1) are typically found to have a blowup set of zero measure. However, the existence of the global term $K^{2}(t)$ in problem (1.2) leads to a nonlocal form of blowup, so that $|u(x, t)|$ tends to infinity as $t \rightarrow T$ at all points on the interval $[0,1]$. We refer to this as nonuniform global blowup although $u(x, t)$ blows up everywhere in the domain. There are points in the interval where the rate of blowup is more rapid than elsewhere in the domain. (We reserve the term uniform global blowup for systems in which $u(x, t) \rightarrow \infty$ at a similar rate for all points of the domain. We consider systems that behave in this manner in a subsequent paper.) Other examples of nonlocal blowup are given in [2] and [16].

In this paper, we establish the following results on the asymptotic behaviour of the function $u(x, t)$ satisfying (1.2)-(1.4).

THEOREM 1.1. (i) The problem has a zero steady state and, for each positive integer $m$, has precisely two nonzero steady-state solutions with $m$ zeros on the interval $[0,1]$.

(ii) There is a constant $C$ such that, if $\left\|u_{0}\right\|_{L_{2}}<C$, then both

$$
\|u(x, t)\|_{H^{1} \rightarrow 0} \text { and }\|u(x, t)\|_{L_{\infty} \rightarrow 0} \quad \text { as } t \rightarrow \infty \text {. }
$$

(iii) If $\int_{0}^{1} u_{0}(x) \cos (n \pi x) d x>0$ for all $n>0$ and if $\int_{0}^{1} u_{0}(x) \cos (\pi x) d x>$ $\int_{0}^{1} u_{0}(x) \cos (2 \pi x) d x$, then, if

$$
\int_{0}^{1} u_{0}(x) \cos (2 \pi x) d x>4 \pi^{2}
$$

or if

$$
\int_{0}^{1} u_{0}(x) \cos (\pi x) d x>4 \sqrt{2} \pi^{2}
$$

then

$$
u(0, t) \text { and }\|u(x, t)\|_{L_{2}}
$$

both tend to infinity at a finite time $T$.

Thus, for small initial data, $u(x, t) \rightarrow 0$, whereas, for suitable larger data, it blows up in a finite time. We show by numerical examples, that the above sufficient conditions 
on blowup are by no means tight. In addition, if we presume that a blowing up solution has a single peak at $x=0$, then we may establish the following asymptotic description of its behaviour as $t \rightarrow T$.

Proposition 1.2. (i) As $t \rightarrow T$, then, if

$$
0 \leqq x<O\left((T-t)^{1 / 2}|\log (T-t)|^{1 / 2}\right),
$$

then

$$
\frac{1}{u(x, t)} \sim T-t+\left(1+\frac{5}{4} \frac{\log (\xi)}{\xi}\right) \frac{x^{2}}{8 \xi}+O\left((T-t) \xi^{-1}\right)
$$

where

$$
\xi=\alpha-\log (T-t) \rightarrow \infty \quad \text { as } t \rightarrow T^{-},
$$

and $\alpha$ is a constant determined both by the initial conditions and the effects of the integral constraint.

(ii) Away from the point of blowup at $x=0, u(x, t)$ takes on the following basic asymptotic structure:

$$
\begin{aligned}
u(x, t) \sim & {\left[T-t+\left(1+\frac{1}{4} \frac{\log (\lambda)}{\lambda}\right) \frac{x^{2}}{8 \lambda}+O\left(x^{2}\right)\right]^{-1} } \\
& -\frac{\sqrt{2} \pi}{(T-t)^{1 / 2}}|\log (T-t)|\left[1-\frac{5}{8} \frac{\log |\log (T-t)|}{|\log (T-t)|}\right]+O\left((T-t)^{-1 / 2}\right),
\end{aligned}
$$

where $\lambda=\alpha-\log \left(x^{2} / 8\right)$. Thus $u(x, t)$ exhibits nonuniform global blowup over the interval $[0,1]$ and is nearly constant in space for much of this interval.

(iii) The function $u(x, t)$ has a zero $x_{*}$ such that

$$
\begin{aligned}
x_{*}^{2} \sim & \frac{2 \sqrt{2}}{\pi}(T-t)^{1 / 2}|\log (T-t)|^{1 / 2} \\
\cdot & {\left[1-\frac{7}{8} \frac{\log |\log (T-t)|}{|\log (T-t)|}+O\left(\frac{1}{\log (T-t)}\right)\right] . }
\end{aligned}
$$

We schematically illustrate the form of $u(x, t)$ close to blowup in Fig. 1.1.

It is interesting to compare the conclusions of Theorem 1.1 and Proposition 1.2 with results on the chemotaxis equations presented in [6] and [7]. This system describes the evolution of a collection of cells in a fixed domain for which a change in the

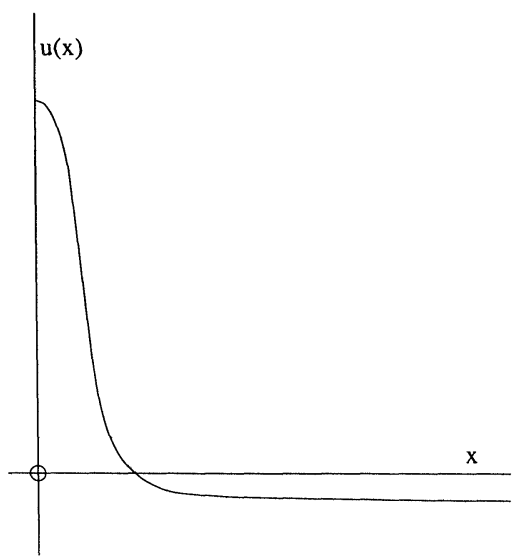

FIG. 1.1. Schematic of the shape of the function $u(x, t)$ close to blowup. 
chemical concentration of the medium containing the cells can result in cell clustering. It is observed experimentally that the cells can form aggregates of high density in a process called chemotactic collapse. This process can be modelled by a system of two parabolic partial differential equations (PDEs) describing the cell and chemical concentrations, with Neumann boundary conditions and a quadratic nonlinearity. In this system, the cell density becomes infinite (blows up) in a finite time, although the integral of the cell density over space (the total cell mass) is conserved. By using asymptotic techniques, it is demonstrated in [6] that blowup occurs if the total mass of cells exceeds a critical value and that the initial density profile is sufficiently concentrated. These results are similar to the conclusions of this paper and indicate that blowup can occur in many systems with conserved quantities.

We present some numerical calculations that support the asymptotic formulae in Proposition 1.2 and show that the asymptotic behaviour close to blowup is independent of the initial conditions. In contrast, the blowup time $T$ depends critically upon $u_{0}(x)$, and, for large values of $u_{0}(0)$, we may estimate it roughly from (1.7) to be $T \sim 1 / u_{0}(0)$, as for the ordinary differential equation (ODE) $d u / d t=u^{2}$.

We may compare the formulae in Proposition 1.2 with expressions derived by Dold [8] and Galaktionov [13] that describe the blowup of the following unconstrained system:

$$
v_{t}=v_{x x}+v^{2}, \quad v_{x}(0)=v_{x}(1)=0, \quad v(0, x)=v_{0}(x) .
$$

It is shown in [3] and [11] that, for suitable initial conditions as $t \rightarrow S$ where $S$ is the blowup time, this system blows up at the single point $x=0$. Close to $x=0, v(x, t)$ has a very similar form to expression (1.7), although it appears, from numerical evidence, that the corresponding value of $\alpha$ tends to be higher for this unconstrained problem.

In Fig. 1.2, we demonstrate this by presenting a numerical calculation of the form of $u(x, t), v(x, t)$ for problems (1.2), (1.4), and (1.11), respectively, (the details of the

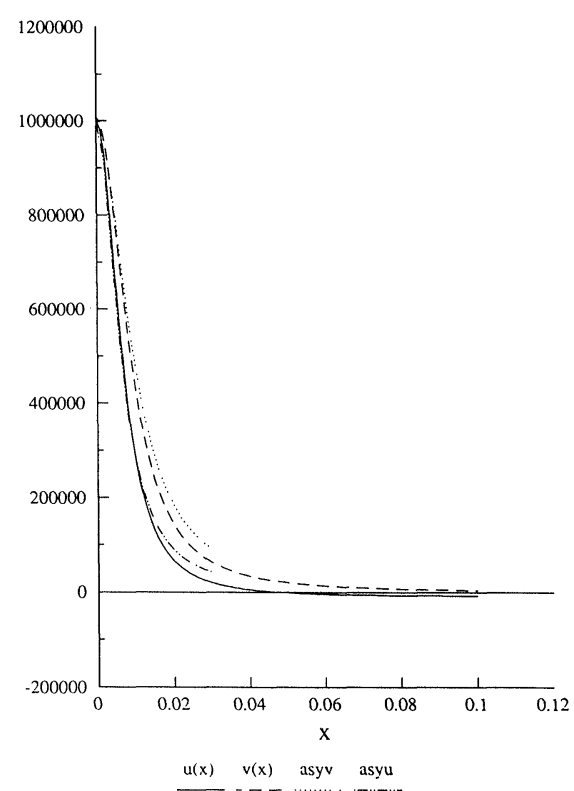

FIG. 1.2. Comparison of the profiles of the constrained and unconstrained problems together with two asymptotic profiles. 
numerical scheme are given in $\S 6)$. In this calculation, we use the initial data $u_{0}(x)=$ $100 \cos (\pi x)$ and $v_{0}(x)=100 \cos (\pi x)$. The corresponding blowup times $T, S$ are, respectively, $T=0.0171 \ldots$ and $S=0.0111 \ldots$ In the figures, we stop the calculations at times $t_{1}, t_{2}$ so that

$$
u\left(0, t_{1}\right)=v\left(0, t_{2}\right)=10^{6} .
$$

We compare the functions $u\left(x, t_{1}\right), v\left(x, t_{2}\right)$ with plots of two of the functions described by formulae (1.7), (1.8) labelled $\operatorname{asyu}(x)$ and $a s y v(x)$ in which we take $\alpha=-9$ and $\alpha=0$. We find that the value $\alpha=0$ gives a good fit for $v(x, t)$ and that a lower value is required to accurately model $u(x, t)$.

These figures demonstrate that, as well as causing the function $u(x, t)$ to exhibit nonuniform global blowup, the effect of the integral constraint is to sharpen the shape of its peak in comparison to the peak of $v(x, t)$ and to increase the blowup time.

The layout of this paper is as follows. In $\S 2$ we study the nonzero steady solutions of problem (1.2), (1.4) and characterise these in terms of their number of zeros. In $\S 3$ we show that the zero steady solution is stable and, by numerically computing the normal mode growth rates of the nonzero steady states, show that these are unstable. The resulting evolution of $u(x, t)$ is then calculated numerically. In $\S 4$ we establish that, for suitable $u_{0}(x), u(x, t)$ does blow up in a finite time $T$. In $\S 5$ we examine the asymptotic structure of $u(x, t)$ close to the blowup time and establish the formulae in Proposition 1.2. Finally, in $\S 6$ we present some numerical calculations supporting Proposition 1.2. It is well known [3] that such numerical calculations are difficult, because the asymptotic structure described in (1.7) only becomes apparent when $|\log (T-t)|$ is large, and, for these values of $t, u(x, t)$ is very large indeed. Consequently, the numerical calculation is prone to errors. We overcome this by using a systematic remeshing of the interval $[0,1]$ as $t \rightarrow T$. Our numerical approach is similar in spirit to the algorithm described in [3], but uses much less a priori information about the solution and hence is applicable to a wider class of problems. The close agreement we find between the asymptotic formulae and the numerical calculations serves to justify both our asymptotic and numerical approaches.

2. The existence of steady-state solutions. In this section, we prove the existence of infinitely many nonzero steady-state solutions of problem (1.2), (1.4). These solutions satisfy the ODE

$$
u_{x x}+u^{2}-K^{2}=0, \quad u_{x}(0)=u_{x}(1)=0, \quad \int_{0}^{1} u(x) d x=0 .
$$

LEMMA 2.1. For each integer $m>0$, problem (2.1) has precisely two nonzero solutions $u_{m}(x)$ and $v_{m}(x)$ such that $u_{m}(0)>0>v_{m}(0)$, and both $u_{m}$ and $v_{m}$ have precisely $m$ zeros in the interval $(0,1)$.

Moreover, if $x<1 / m$, then $u_{m}(x)=m^{2} u_{1}(m x)$ and $v_{m}(x)=m^{2} v_{1}(m x)$.

Proof. To establish the existence of these solutions, we use rescaling and phaseplane arguments similar to those used in [21].

Suppose that the function $w(s)$ is a solution of the following differential equation problem:

$$
\begin{gathered}
w_{s s}+w^{2}-1=0, \\
w_{s}(0)=w_{s}\left(K^{1 / 2}\right)=0, \\
\int_{0}^{K^{1 / 2}} w(s) d s=0 .
\end{gathered}
$$


By setting

$$
u(x)=K w\left(K^{1 / 2} x\right),
$$

a simple calculation shows that $u(x)$ is a solution of problem (2.1). We may exactly integrate (2.2) to deduce that any solution of (2.2) satisfies the following identity:

$$
\frac{1}{2} w_{s}^{2}+\frac{1}{3} w^{3}-w=C-\frac{2}{3} \text {. }
$$

Here $C$ is a constant. From this identity, we can see that (2.2) has a series of bounded, periodic solutions parameterised by $C$. Indeed, in the phase plane $\left(w, w_{s}\right)$, these solutions lie on a series of closed curves. These curves enclose the stable centre $\left(w, w_{s}\right)=(1,0)$ for which $C=0$ and are bounded by a homoclinic orbit $\Gamma$, which includes the unstable saddle point $(-1,0)$ so that $C=4 / 3$. It is easy to show that $\Gamma$ intersects the line $w_{s}=0$ at the point $(2,0)$, and a further calculation shows that it corresponds to the exact solution $w_{H}(s)=2-3 \tanh ^{2}(s / \sqrt{2})$ of problem (2.2). We illustrate the form of the phase plane in Fig. 2.1.

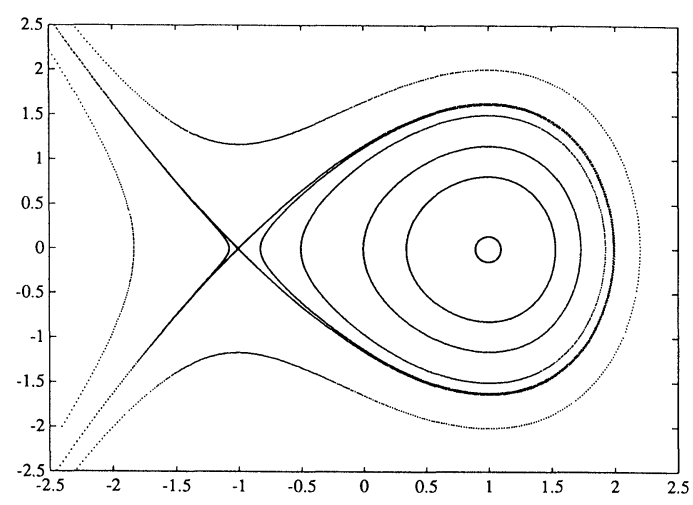

FIG. 2.1. Phase plane trajectories satisfied by solutions of the steady-state equation.

A solution of problem (2.2), which satisfies the boundary conditions (2.3), corresponds to a trajectory in the phase plane that intersects the $w^{\prime}=0$ axis. To satisfy the integral constraint (2.4), such a trajectory must also intersect the line $w=0$. It also follows that, from the symmetry of the differential equation (2.2), such a trajectory is symmetric about the line $w^{\prime}=0$. Let a solution of $(2.2),(2.3)$ correspond to a trajectory that intersects the line $w^{\prime}=0$ at the point $A=(\gamma, 0), 1<\gamma<2$, when $s=0$, and then intersects the line $w^{\prime}=0$ again at a first point $B=(-\delta, 0), 0<\delta<1$ when $s=K_{1}^{1 / 2}$. That is, we consider a solution of (2.2), (2.3) involving only a single transition from $A$ to $B$ on an orbit in the negative half plane $w^{\prime} \leqq 0$. The function $w(s)$ is then a continuous function of $\gamma$. Hence, both $K_{1}$ and the integral

$$
I(\gamma) \equiv \int_{0}^{K_{1}^{1 / 2}} w(s, \gamma) d s
$$

are also continuous functions of $\gamma$. If $\gamma$ is close to 1 , then $w(s)$ is a small perturbation of the steady solution $w \equiv 1$ so that

$$
w(s) \approx 1+(\gamma-1) \cos \sqrt{2} s .
$$

Thus, $K_{1}^{1 / 2} \approx \pi / \sqrt{2}$ and $I(\gamma) \approx \pi / \sqrt{2}>0$. Similarly, if $\gamma$ is close to 2 , then $w(s)$ is "close" to $w_{H}(s)$ in the sense that, on any compact interval, $w(s) \rightarrow w_{H}(s)$ as $\gamma \rightarrow 2$. 
We may deduce that, as $\gamma \rightarrow 2$, then $K_{1} \rightarrow \infty$ and $I(\gamma) \rightarrow-\infty$. It follows that there is at least one value of $\gamma$ such that $1<\gamma<2$ for which $I(\gamma)=0$. It is shown further in [22] that $\gamma$ is unique. For this value of $\gamma$, the function $w(s)$ satisfies all the conditions (2.2)-(2.4). Furthermore, $w(0)>0$ and $w(s)$ has precisely one zero in the interval $[0,1]$. We may similarly construct another solution with one zero by taking the portion of the trajectory that lies between $B$ and $A$ in the upper half plane for which $w^{\prime}(s)>0$. We denote these two solutions as $w^{+}(s)$ and $w^{-}(s)$, respectively. It is clear from the symmetry of the system that $w^{+}(s)=w^{-}\left(K_{1}^{1 / 2}-s\right)$. We now rescale the solutions so that

$$
u_{1}(x)=K_{1} w^{+}\left(K_{1}^{1 / 2} x\right) \text { and } v_{1}(x)=K_{1} w^{-}\left(K_{1}^{1 / 2} x\right) \text {. }
$$

Further solutions may be constructed from these two basic solutions by reflecting and rescaling. We may set $K=m^{2} K_{1}$, and, for example,

$$
\begin{aligned}
& u_{2}(x)=4 u_{1}(2 x) \quad \text { if } 0 \leqq x \leqq \frac{1}{2}, \quad u_{2}(x)=4 v_{1}\left(2\left(x-\frac{1}{2}\right)\right) \quad \text { if } \frac{1}{2} \leqq x \leqq 1, \\
& v_{2}(x)=4 v_{1}(2 x) \quad \text { if } 0 \leqq x \leqq \frac{1}{2}, \quad v_{2}(x)=4 u_{1}\left(2\left(x-\frac{1}{2}\right)\right) \quad \text { if } \frac{1}{2} \leqq x \leqq 1 \text {, }
\end{aligned}
$$

with similar constructions for $u_{m}(x)$ and $v_{m}(x)$.

The proof of this result leads to a numerical algorithm for calculating the functions $u(x)$ and $v(x)$, which gives the following values for the above constants:

$$
K_{1}^{1 / 2}=4.2483679, \quad w^{+}(0)=1.9988307, \quad w^{-}(0)=-0.94032 .
$$

The resulting functions have the form illustrated in Fig. 2.2.

3. The stability of the zero steady state and the evolution of the solution from the nonzero steady states. We have now shown that problem (1.2), (1.4) has a sequence of nonzero steady-state solutions as well as a zero steady state. In this section, we prove that the zero steady state is stable to small perturbations. We also demonstrate by numerically computing their unstable eigenmodes that the nonzero steady-state solutions are unstable. We determine numerically the resulting evolution of the solution as it blows up in a finite time. Finally, we also consider the evolution of the function $u(x, t)$ from a variety of initial data and determine a threshold for blowup to occur.

The stability of the zero solution of the unconstrained problem (1.1) or the existence of solutions that blow up in finite time is often proved by using the maximum principle in the following form: If $u(x, t)$ is a solution of problem (1.1) with initial data $u_{0}(x)$ and if $v(x, t)$ is another solution with initial data $v_{0}(x)$, then, if $u_{0}(x)<v_{0}(x)$ for all $x \in(0,1)$, then $u(x, t)<v(x, t)$ for all $x \in(0,1)$ and for all $t$ such that $u(x, t)$ and $v(x, t)$ exist as bounded functions. The stability (or finite-time blowup) of a positive solution may then be proved by bounding it above (or below) by a known stable (or unstable) solution.

This form of the maximum principle cannot be applied easily to the constrained problem (1.2), (1.4) because, if $u_{0}(x)<v_{0}(x)$, then, when we consider their integral, it follows that

$$
0=\int_{0}^{1} u_{0}(x) d x<\int_{0}^{1} v_{0}(x) d x=0,
$$

which is a contradiction. Thus, $u_{0}(x)$ and $v_{0}(x)$ must intersect at one point at least. Instead of using the maximum principle, we prove the stability of the zero solution of (1.2), (1.4) by using an energy argument.

LeMmA 3.1. (i) Suppose that $u(x, t)$ is a solution of problem (1.2), (1.4) such that $u(x, t) \in H^{1}(0,1)$; then there is a constant $C>0$ such that, if $\left\|u_{0}\right\|_{L_{2}}<C$, then $\|u\|_{L_{2}} \rightarrow 0$ as $t \rightarrow \infty$. 


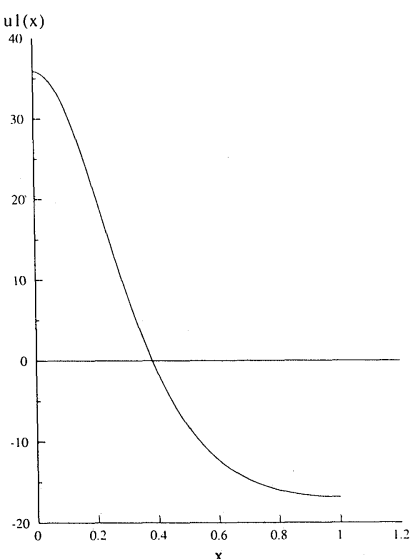

(a)

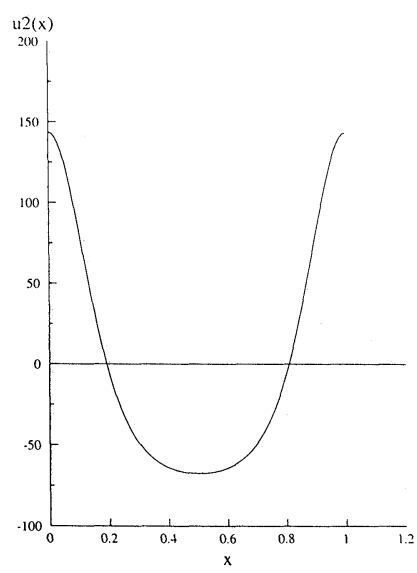

(b)

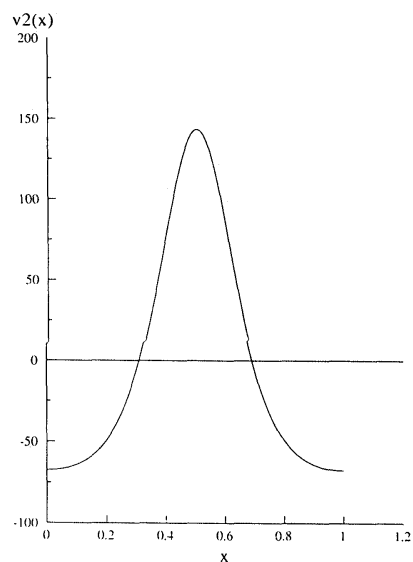

(c)

FIG. 2.2. Forms of the steady-state solutions (a) $u_{1}(x)$, (b) $u_{2}(x)$, and (c) $v_{2}(x)$. 
(ii) If, furthermore, $u(x, t) \in H^{2}(0,1)$, then $\|u\|_{H^{1} \rightarrow 0}$ as $t \rightarrow \infty$, and hence $\|u\|_{\infty} \rightarrow 0$ as $t \rightarrow \infty$.

Proof. If we multiply (1.2) by $u$ and integrate with respect to $x$, it follows that

$$
\frac{1}{2} \frac{d}{d t}\|u\|_{L_{2}}^{2}=\int_{0}^{1} u u_{x x} d x+\int_{0}^{1} u^{3} d x-\int_{0}^{1} u d x \int_{0}^{1} u^{2} d x
$$

We may integrate this by parts to deduce that

$$
\frac{1}{2} \frac{d}{d t}\|u\|_{L_{2}}^{2}=-\int_{0}^{1} u_{x}^{2} d x+\int_{0}^{1} u^{3} d x
$$

It follows from Hölders inequality that

$$
\int_{0}^{1} u^{3} d x<\left(\int_{0}^{1} u^{2} d x\right)^{1 / 2}\left(\int_{0}^{1} u^{4} d x\right)^{1 / 2}=\|u\|_{L_{2}}\|u\|_{L_{4}}^{2} .
$$

As $\int_{0}^{1} u d x=0$, we may deduce from the form of the Sobolev imbedding theorem quoted in [4] that

$$
\|u\|_{L_{4}}^{2}<\frac{1}{C} \int_{0}^{1} u_{x}^{2} d x
$$

where $C>0$ is a constant. Combining these inequalities, we have

$$
\frac{1}{2} \frac{d}{d t}\|u\|_{L_{2}}^{2}<\left(\int_{0}^{1} u_{x}^{2} d x\right)\left(-1+\frac{1}{C}\|u\|_{L_{2}}\right)
$$

Moreover, the Poincaré inequality combined with the condition $\int_{0}^{1} u d x=0$ again implies that there is a constant $A>0$ such that

$$
\|u\|_{L_{2}}^{2}<A \int_{0}^{1} u_{x}^{2} d x
$$

Combining these inequalities, we may deduce that, if $\|u\|_{L_{2}}<C$, then

$$
\frac{1}{2} \frac{d}{d t}\|u\|_{L_{2}}^{2}<\frac{-1}{A C}\left(C-\|u\|_{L_{2}}\right)\|u\|_{L_{2}}^{2} \text {. }
$$

Thus, $\|u\|_{L_{2}} \rightarrow 0$ as $t \rightarrow \infty$. This proves (i).

To prove (ii), we first differentiate (1.2) with respect to $x$ to give

$$
u_{x t}=u_{x x x}+2 u u_{x} \text {. }
$$

Multiplying by $u_{x}$ and integrating with respect to $x$ gives

$$
\frac{1}{2} \frac{d}{d t} \int u_{x}^{2} d x=-\int_{0}^{1} u_{x x}^{2} d x+2 \int_{0}^{1} u u_{x}^{2} d x
$$

where we have made use of the assumption that $u$ is sufficiently smooth for the above integrals to exist. It follows from Hölders inequality and the Sobolev imbedding theorems that there is a constant $D$ such that

$$
\frac{1}{2} \frac{d}{d t}\left\|u_{x}\right\|_{L_{2}}^{2} \leqq-\left\|u_{x x}\right\|_{L_{2}}^{2}+2\|u\|_{L_{2}}\left\|u_{x}\right\|_{L_{4}}^{2} \leqq-\left\|u_{x x}\right\|_{L_{2}}^{2}+\frac{2}{D}\|u\|_{L_{2}}\left\|u_{x x}\right\|_{L_{2}}^{2} .
$$

Thus

$$
\frac{1}{2} \frac{d}{d t}\left\|u_{x}\right\|_{L_{2}}^{2} \leqq-\left\|u_{x x}\right\|_{L_{2}}^{2}\left(1-\frac{2}{D}\|u\|_{L_{2}}\right) .
$$

We now suppose that

$$
\|u\|_{L_{2}}^{2} \leqq \min \left(\frac{D}{2}-\varepsilon \frac{D}{2}, C\right)
$$


for some fixed $\varepsilon>0$. It then follows from the Poincare inequality that there is a further constant $E>0$ such that

$$
\frac{1}{2} \frac{d}{d t}\left\|u_{x}\right\|_{L_{2}}^{2} \leqq-\varepsilon\left\|u_{x x}\right\|_{L_{2}}^{2} \leqq-\frac{E}{2}\left\|u_{x}\right\|_{L_{2}}^{2}
$$

Thus $\left\|u_{x}\right\|_{L_{2}}^{2} \rightarrow 0$ as $t \rightarrow \infty$, and hence $\|u\|_{\infty} \rightarrow 0$. This proves (ii)

Having proved that the zero steady solution of (1.2), (1.4) is stable to small perturbations, we now demonstrate that the nonzero steady states are unstable. To do this, we consider a small time-dependent perturbation to the steady solutions $u_{m}(x)$ and $v_{m}(x)$, which takes the form $\varepsilon e^{\lambda m^{2} t} \psi(x)$ for $\varepsilon \ll 1$. Similarly, we consider a perturbation of the form $\varepsilon m^{2} e^{\lambda m^{2} t} J$ to the constant $K^{2}$. To leading order, $\psi$ and $J$ satisfy the following ODE:

$$
\psi_{x x}+2 w(x) \psi-m^{2} J=\lambda m^{2} \psi, \quad \psi_{x}(0)=\psi_{x}(1)=0, \quad \int_{0}^{1} \psi(x) d x=0,
$$

where $w(x)$ represents either $u_{m}(x)$ or $v_{m}(x)$. It is immediate that

$$
m^{2} J=\int_{0}^{1} 2 w(x) \psi(x) d x .
$$

Without loss of generality, we set

$$
\psi(0)=1 .
$$

We observe that, if $n$ is large, then (3.1) has the following approximate solution:

$$
\psi(x) \approx \cos (n \pi x), \quad m^{2} \lambda \approx-n^{2} \pi^{2}+0(1),
$$

and $J \rightarrow 0$ as $n \rightarrow \infty$.

These solutions correspond to stable time-dependent perturbations of the functions $u_{K}(x)$ and $v_{K}(x)$. For smaller values of $n$, the behaviour is more subtle and depends upon the value of $m$. As further analysis is difficult, we present the results of some numerical calculations of $\lambda$ and $\psi$ for $u_{1}, v_{1}, u_{2}$, and $v_{2}$.

(i) $u_{1}$

\begin{tabular}{|l|l|l|l|}
\hline$\lambda$ & 16.82 & -44.5 & -92.15 \\
\hline$J$ & 15.32 & -6.9 & -1.5 \\
\hline
\end{tabular}

(ii) $v_{1}$

\begin{tabular}{|c|c|c|c|}
\hline$\lambda$ & 16.82 & -44.5 & -92.15 \\
\hline$J$ & -50.0 & -5.12 & 1.1 \\
\hline
\end{tabular}

(iii) $u_{2}$

\begin{tabular}{|c|c|c|}
\hline$\lambda$ & 45.1 & 16.82 \\
\hline$J$ & 0 & 15.32 \\
\hline
\end{tabular}

(iv) $v_{2}$

\begin{tabular}{|l|l|l|}
\hline$\lambda$ & 16.82 & 0.05 \\
\hline$J$ & -50.0 & 0 \\
\hline
\end{tabular}


When $m=1$, the eigensolutions for $u_{1}(x)$ and $v_{1}(x)$ are related by the transformation $x \rightarrow 1-x$ followed by a simple rescaling of $\psi(x)$. We see that there is precisely one unstable eigenmode, which we denote by $\psi_{1}(x)$, for the function $u_{1}(x)$. Numerical computations demonstrate that, if we take initial data $u_{0}(x)=u_{1}(x)-\varepsilon \psi_{1}(x)$ where $\varepsilon>0$, then the corresponding solution of (1.2), (1.4) satisfies $|u(x, t)| \rightarrow 0$ as $t \rightarrow \infty$. Conversely, if we take initial data $u_{0}(x)=u_{1}(x)+\varepsilon \psi_{1}(x)$, then there is a finite time $T$ such that $|u(x, t)| \rightarrow \infty$ as $t \rightarrow T$.

In Fig. 3.1, we present some numerical computations of the form of this solution when $\varepsilon=0.01$. Our figure gives the form of $u(x, t)$ for values of $t \rightarrow T$. It is clear from this that the function $u(x, t)$ develops a pronounced positive spike at the point $x=0$, which is similar in form to the solution of problem (1.1). Furthermore, $u(1, t)<0$ and $u(1, t) \rightarrow-\infty$, but at a much slower rate than $u(0, t)$. In $\S 5$ we study the asymptotic form of this blowing-up solution.

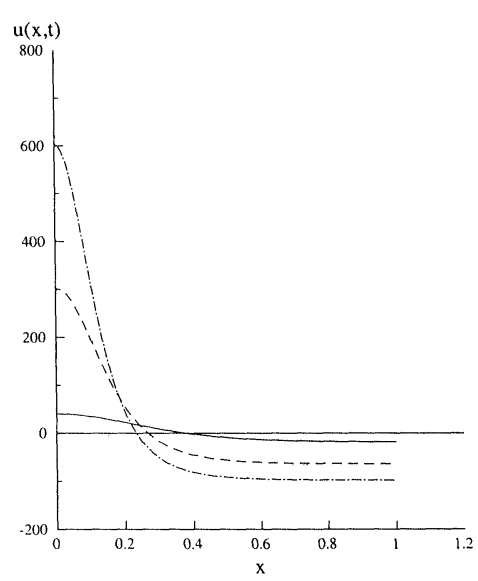

FIG. 3.1. Blowup in which the steady state $u_{1}(x)$ is perturbed by the most unstable eigenfunction.

When $m=2$, we observe different behaviour for the two steady states $u_{2}(x)$ and $v_{2}(x)$. There are two unstable eigensolutions $\psi_{2}(x)$ and $\psi_{3}(x)$ for $u_{2}(x)$ with corresponding eigenvalues $\lambda_{3}>\lambda_{2}>0$. The function $\psi_{2}(x)$ is symmetric about the point $x=\frac{1}{2}$ and is a rescaled form of $\psi_{1}(x)$ such that, if $x<\frac{1}{2}, \psi_{2}(x)=\psi_{1}(2 x)$ and, if $x>\frac{1}{2}, \psi_{2}(x)=$ $\psi_{1}(2(1-x))$. In contrast, the function $\psi_{3}(x)$ is antisymmetric about $x=\frac{1}{2}$ such that $\psi_{3}(x)=-\psi_{3}(1-x)$. As $\lambda_{3}>\lambda_{2}$, we observe that, if we take arbitrary initial data close to $u_{2}(x)$, then $u(x, t)$ tends to evolve in an antisymmetric manner. We demonstrate this numerically by taking an initial condition

$$
u_{0}(x)=u_{2}(x)+0.01 \cos (\pi x) .
$$

The resulting evolution of the function $u(x, t)$ is presented in Fig. 3.2. We can see from these figures that $u(x, t)$ rapidly loses its symmetry about $x=\frac{1}{2}$ and, in its subsequent evolution, develops a positive spike at $x=0$ just as in the case when $m=1$.

In contrast, when we study the steady state $v_{2}(x)$, we see that there are again two unstable eigensolutions $\psi_{4}(x)$ and $\psi_{5}(x)$ so that $\lambda_{4}>\lambda_{5}>0$. In this case, the function $\psi_{4}(x)$ is symmetric about $x=\frac{1}{2}$ so that $\psi_{4}(x)=\psi_{1}(1-2 x)$ if $x<\frac{1}{2}$ and $\psi_{4}(x)=\psi_{4}(1-x)$. Similarly, the function $\psi_{5}(x)$ is antisymmetric. Thus, we expect that, if we take arbitrary initial data close to $v_{2}(x)$, then $u(x, t)$ tends to evolve in a symmetric manner. We demonstrate this by taking an initial condition

$$
u_{0}(x)=v_{2}(x)+0.01 \cos (\pi x) .
$$




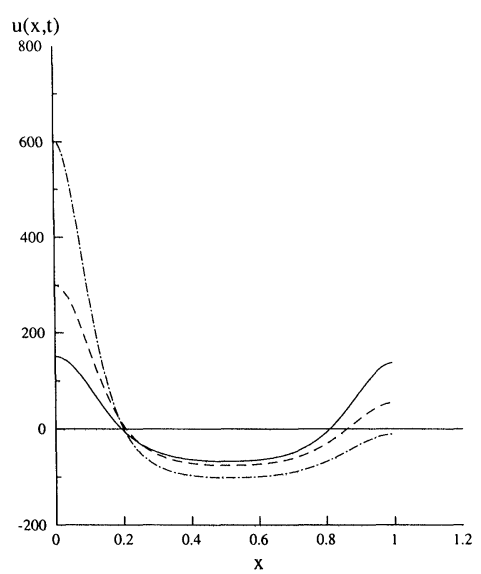

FIG. 3.2. Blowup in which a symmetric initial state with peaks at $x=0$ and $x=1$ loses symmetry.

The resulting evolution is presented in Fig. 3.3. It is clear from this figure that the solution stays symmetric about $x=\frac{1}{2}$ and blows up at $x=\frac{1}{2}$. For higher values of $m$, we conjecture that both $u_{m}(x)$ and $v_{m}(x)$ have $m$-dimensional unstable manifolds.

We conclude this section by studying the evolution of $u(x, t)$ for initial data, which takes the form

$$
u_{0}(x)=\gamma \cos (\pi x), \quad \gamma>0 .
$$

We find numerically that there exists a constant $\gamma^{*} \approx 29.9$ such that

(i) If $\gamma<\gamma^{*}$, then $\|u(x, t)\|_{\infty} \rightarrow 0$ as $t \rightarrow \infty$,

(ii) If $\gamma>\gamma^{*}$, then there exists a time $T(\gamma)$ such that

$$
\|u(x, t)\|_{\infty} \rightarrow \infty \quad \text { as } t \rightarrow T(\gamma) .
$$

In Fig. 3.4, we present a graph of the function $T(\gamma)$. Thus, $\gamma^{*}$ is a critical value dividing the blowing-up and the stable behaviour. (This value is not far from the maximum value of $u_{1}(x)$, which is 36.07 .) If we take initial data

$$
u_{0}(x)=\cos (\pi x)+200 \cos (2 \pi x),
$$

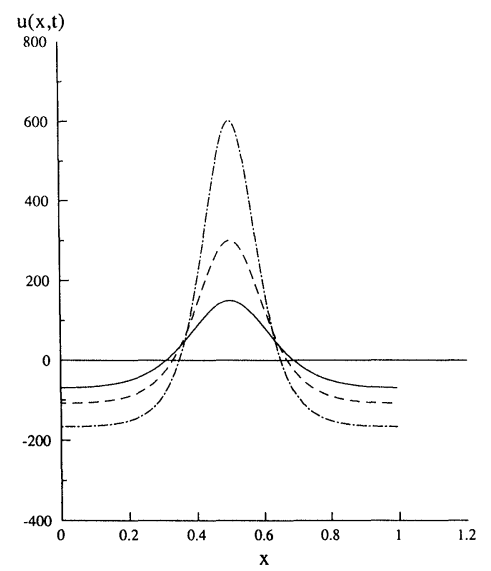

FIG. 3.3. Blowup in which a symmetric initial state with a single peak at $x=\frac{1}{2}$ remains symmetric. 


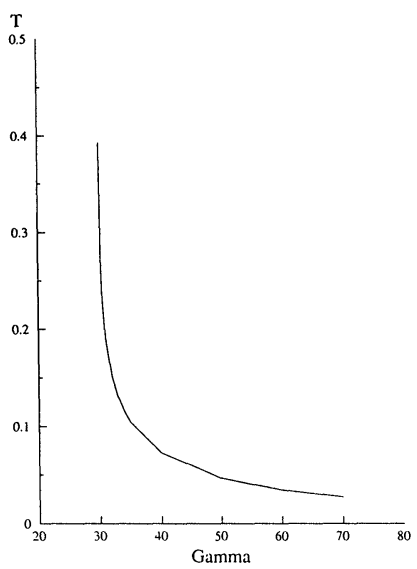

FIG. 3.4. Plot of the blowup time T compared to the magnitude $\gamma$ of the initial data.

which has positive maxima at $x=0.1$, we observe that the solution $u(x, t)$ rapidly evolves to have a single spike at the point $x=0$. In contrast, if we take

$$
u_{0}(x)=\cos \pi x-200 \cos 2 \pi x,
$$

which has a positive maximum close to $x=\frac{1}{2}$, we observe that $u(x, t)$ blows up at the point $x=\frac{1}{2}$. Thus, the location of the blowup point and the form of the blowup depends crucially upon the nature of the initial data. It also appears that arbitrary initial data (of a sufficiently large amplitude for blowup to occur) tends to evolve into a blowup with a single positive peak either at a boundary or at an interior point.

4. Sufficient conditions for finite-time blowup. We have demonstrated in $\S 3$ that there exist solutions of problem (1.2), (1.4) that blow up in a finite time. In this section, we obtain some sufficient conditions on $u_{0}(x)$ that ensure that blow up occurs.

We are unable to prove blowup by using the eigenfunction approach described in [17], since the first nonzero eigenfunction $\phi(x)$ of the differential operator $-d^{2} / d x^{2}$, which also satisfies the Neumann boundary conditions, is the constant function, and, from the integral constraint (1.4), it is clear that $\int_{0}^{1} \phi(x) u(x) d x$ does not blow up. Similarly, the energy method described by Ball [1] is severely limited by the integral constraint, and we are only able to prove exponential growth in the $L_{2}$ norm (see the Appendix). The underlying reason for the inadequacy of both methods is the lack of a comparison principle for the evolution of the problem in the function space $C[0,1]$. To overcome such difficulties in a different system, Palais [18] introduced a method to prove blowup in Fourier space. We now extend the Palais method to prove that system (1.2), (1.4) blows up in finite time.

LEMMA 4.1. We suppose that $u(x, t)$ is a solution of problem (1.2), (1.4) and that $u_{0}(x)$ satisfies the following conditions:

$$
\begin{gathered}
\int_{0}^{1} u_{0}(x) \cos (n \pi x) d x>0 \quad \forall n, \\
\int_{0}^{1} u_{0}(x) \cos (\pi x) d x=C_{1} \text { and } \int_{0}^{1} u_{0}(x) \cos (2 \pi x) d x=C_{2},
\end{gathered}
$$


with $C_{1}>C_{2}$, and either

$$
C_{2}>4 \pi^{2} \text { or } C_{1}>4 \sqrt{2} \pi^{2} .
$$

Then, $u(x, t)$ blows up in a finite time $T$ such that, as $t \rightarrow T$,

$$
u(0, t) \rightarrow \infty \text { and }\|u\|_{L^{2} \rightarrow \infty} .
$$

Note. The numerical studies presented in $\S 3$ show that blowup occurs if, for example, $C_{2}=0$ and $C_{1}>\gamma^{*} / 2 \approx 14.9$. Indeed, by a simple numerical estimate, we may extend the proof given here to show that blowup must occur if $C_{1}>23.60731$. Thus the sufficient conditions (4.3) may be substantially relaxed.

Proof. Because the function $u(x, t)$ satisfies Neumann boundary conditions, it can be expanded as a Fourier series of the form

$$
u(x, t)=\sum_{n=-\infty}^{\infty} C_{n}(t) e^{\mathrm{in} \pi x},
$$

where $C_{n}(t)=C_{-n}(t)$ for all $t$. The integral constraint (1.4) implies that $C_{0}(t)=0$ for all $t$. Substituting this expression into (1.2) and equating coefficients, we find that for $n \neq 0$

$$
\frac{d}{d t} C_{n}=-n^{2} \pi^{2} C_{n}+\sum_{m=-\infty}^{\infty} C_{m} C_{n-m}
$$

for all times such that a solution exists.

LEMмA 3.2. If $C_{n}(0)>0$ for all $n$, then, for all subsequent times (such that a solution exists),

$$
C_{n}(t)>0 .
$$

Proof. Suppose that $C_{n}$ is the first coefficient to violate (4.6) at a time $t_{*}$ such that $C_{n}\left(t_{*}\right)=0$. It follows from (4.5) that

$$
\frac{d}{d t}\left(e^{n^{2} \pi^{2} t} C_{n}(t)\right)=e^{n^{2} \pi^{2} t} \sum C_{m} C_{n-m} \equiv f(t),
$$

where $f(t)>0$ for $0 \leqq t<t^{*}$. Thus

$$
C_{n}\left(t^{*}\right)=e^{-n^{2} \pi^{2} t_{*}}\left[C_{n}(0)+\int_{0}^{t^{*}} f(t) d t\right]>0,
$$

which is a contradiction.

We now consider the evolution of the two components $C_{1}(t)$ and $C_{2}(t)$. We may deduce from (4.6) that, if conditions (4.1), (4.2) are satisfied, then $C_{1}$ and $C_{2}$ satisfy the following differential inequality:

$$
\frac{d C_{1}}{d t}>-\pi^{2} C_{1}+C_{1} C_{2} \text { and } \frac{d C_{2}}{d t}>-4 \pi^{2} C_{2}+C_{1}^{2}
$$

It is easy to show that since $C_{n}(t)>0$, then, if $p$ and $q$ satisfy the system of differential equations

$$
\frac{d p}{d t}=-\pi^{2} p+p q, \quad \frac{d q}{d t}=-4 \pi^{2} q+p^{2},
$$

and if $C_{1}(0)=p(0)$ and $C_{2}(0)=q(0)$, then

$$
C_{1}(t)>p(t) \quad \text { and } \quad C_{2}(t)>q(t) \quad \forall t>0 .
$$


Thus, to study the blowup of $C_{1}$ and $C_{2}$, we need only study the solutions of (4.8). This system has an attracting node at the point $(p, q)=(0,0)$ and a saddle point $S$ at the point $S \equiv(p, q)=\left(2 \pi^{2}, \pi^{2}\right)$. The point $S$ has an unstable manifold $W_{u}$ and a stable manifold $W_{s}$ such that $W_{s}=\left\{(p, q): q=W_{s}(p)\right\}$. Numerical computations imply that $W_{s}$ intersects the $p$-axis at the point $(23.60731,0)$, and, if $q(0)>W_{s}(p(0))$, then the consequent solution of $(4.8)$ blows up in a finite time. This behaviour is illustrated in Fig. 4.1. To obtain rigorous estimates on blowup, we note that, if $p=q$, then $d p / d t=$ $p\left(p-\pi^{2}\right)$ and $d q / d t=p\left(p-4 \pi^{2}\right)$. Thus, if $p>\pi^{2}$, then $d q / d p<1$. Hence, if at $t=0$, $p>q$, then $p(t)>q(t)$ for all $t>0$, provided that $p(t)>\pi^{2}$.

Suppose now that $p>q>4 \pi^{2}$; then

$$
\frac{d q}{d t}>-4 \pi^{2} q+q^{2}=q\left(q-4 \pi^{2}\right) .
$$

Thus $d q / d t>0$; hence $p(t)>q(t)>4 \pi^{2}$ for all $t>0$. Moreover, a simple integration of the differential inequality (4.9) shows that

$$
1-\frac{4 \pi^{2}}{q(t)}>\left[1-\frac{4 \pi^{2}}{q(0)}\right] e^{4 \pi^{2} t}
$$

Thus, $q(t)$ blows up in a finite time $T$ such that

$$
T<-1 / 4 \pi^{2} \log \left(1-4 \pi^{2} / q(0)\right) .
$$

We conclude that both $C_{1}(t)$ and $C_{2}(t)$ blow up in a finite time if

$$
C_{1}(0)>C_{2}(0)>4 \pi^{2} .
$$

To extend this estimate further, we note that as $p q>0$, then $p(t)>p(0) e^{-\pi^{2} t}$. Thus

$$
\frac{d q}{d t}>-4 \pi^{2} q+p(0)^{2} e^{-2 \pi^{2} t}
$$

so that

$$
q(t)>\frac{p(0)^{2}}{2 \pi^{2}} e^{-2 \pi^{2} t}+\left(q(0)-\frac{p(0)^{2}}{2 \pi^{2}}\right) e^{-4 \pi^{2} t} .
$$

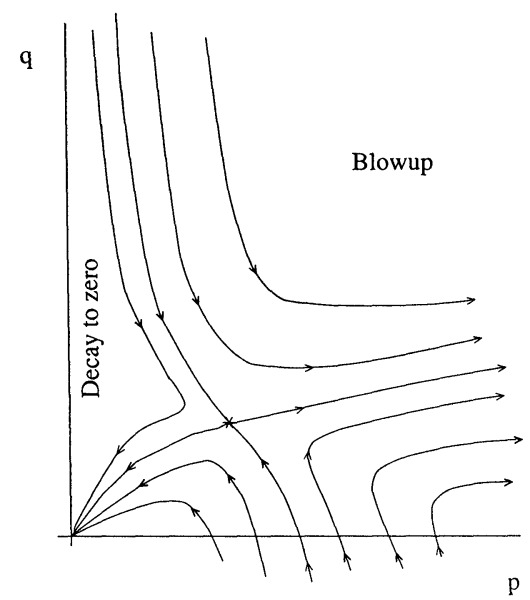

FIG. 4.1. Phase plane of the solution trajectories for the two-mode truncation of the blowup equations. 
Either $q(0)>p(0)^{2} / 2$ or, if $q(0)<p(0)^{2} / 2$, we may maximise this expression over all times $t$ to conclude that there is a time $t^{*}$ such that

$$
q\left(t^{*}\right)>\frac{1}{16 \pi^{4}} \frac{p(0)^{4}}{\left(p(0)^{2} /\left(2 \pi^{2}\right)-q(0)\right)}>\frac{p(0)^{2}}{8 \pi^{2}} .
$$

Thus, without loss of generality, we may assume that, at some time $t^{*}$,

$$
q\left(t^{*}\right)>\frac{p(0)^{2}}{8 \pi^{2}}
$$

Thus, if $p(0)>q(0)$, then, from the previous result, $p(t)>q(t)$ for all $t>0$, and, if $p(0)>4 \sqrt{2} \pi^{2}$ then, at a time $t, q\left(t^{*}\right)>4 \pi^{2}$. Hence, blowup occurs in this case as well.

As $C_{n}(t)>0$ for all $t<T$, it follows immediately that $u(0, t)>u(x, t)$ for all $t<T$. Thus, if $u(0, t)$ is finite, then so is $u(x, t)$ for all $x$. However, as $u(0, t)>C_{1}(t)+C_{2}(t)$, it follows that a solution of (1.2), (1.4) blows up at the origin in a finite time. (Indeed it blows up at all points in the interval $[0,1]$.)

To prove that $\|u\|_{L^{2}}$ also blows up in a finite time, we assume that, in contrast, $\|u\|_{L^{2}}<L$ for all $t \leqq T$. It then follows directly from the definition of $C_{n}$ that

$$
\frac{d}{d t} C_{n} \leqq-n^{2} \pi^{2} C_{n}+L^{2}
$$

Integrating this inequality, we have

$$
C_{n}(t) \leqq C_{n}(0) e^{-n^{2} \pi^{2} t}+\frac{L^{2}}{n^{2} \pi^{2}} .
$$

Thus, summing the resulting series, we deduce that

$$
u(0, t)=\sum_{-\infty}^{\infty} C_{n}(t) \leqq u(0,0) e^{-n^{2} \pi^{2} t}+\frac{L^{2}}{6} .
$$

However, this contradicts the fact that $u(x, t)$ blows up at the origin. We conclude that $L$ cannot be finite, and hence $\|u\|_{L^{2} \rightarrow \infty}$ as $t \rightarrow T$.

5. An asymptotic description of the blowup of $u(x, t)$. In this section, we obtain an asymptotic description of the function $u(x, t)$ close to the blowup time $T$ on the assumption that the blowup is most pronounced at the point $x=0$.

Our numerical studies, described in $\S 3$, showed that, for certain forms of initial data (for example, $u_{0}(x)=\gamma \cos \pi x$ ), the solution develops a pronounced positive spike at the point $x=0$ away from which it is approximately constant in space and takes a negative value. The spike grows in magnitude and becomes narrower as $t \rightarrow T$, such that, if $x^{*}$ is the first point so that $u\left(x^{*}, t\right)=0$, then $x^{*} \rightarrow 0$ as $t \rightarrow T$. The solution also exhibits nonuniform global blowup such that

$$
|u(x, t)| \rightarrow \infty \quad \text { as } t \rightarrow T \quad \forall x \in[0,1]
$$

but the rate of blowup is most pronounced close to $x=0$.

We now construct an asymptotic description of the blowup profile by rescaling the solution inside the spike. We first consider the unconstrained problem (1.11), together with the boundary conditions

$$
v_{x}(0)=0
$$

As we have demonstrated numerically, the solutions of this problem can blow up at the origin in finite time. When this occurs, $v(0, t) \sim(T-t)^{-1}$, and the function $u(x, t)$ develops a spike at the origin of width $O\left[(T-t)^{1 / 2}\right]$ apart from a relatively weak factor of $|\log (T-t)|^{1 / 2}$. Following the approach to this problem described in [3], we introduce 
similarity variables motivated by these observations to study problem (1.2). Accordingly, we set

$$
\zeta=\frac{x}{\sqrt{(T-t)}}, \quad s=-\log (T-t), \quad \text { and } \quad W(\zeta, S)=(T-t) u(x, t),
$$

where $T$ is the blowup time and $s \rightarrow \infty$ as $t \rightarrow T$. Then the function $u(x, t)$ is a solution of (1.2), (1.4) if the function $W(\zeta, s)$ is a solution of the system

$$
W_{s}-W_{\zeta \zeta}+\frac{\zeta}{2} W_{\zeta}+W-W^{2}+e^{-s / 2} \int_{0}^{e^{s / 2}} W^{2} d \zeta=0 .
$$

If we make a similar substitution of variables into (1.1), we obtain, in contrast, the system without an integral term

$$
W_{s}-W_{\zeta \zeta}+\frac{\zeta}{2} W_{\zeta}+W-W^{2}=0
$$

It is well known [3], [8] that problem (5.4) has a solution $W^{0}(\zeta, S)$ such that

$$
W^{0}(\zeta, s)=0\left(\frac{1}{1+\zeta^{2} / s}\right) \text { as } s \rightarrow \infty
$$

Thus $e^{-s / 2} \int_{0}^{\infty}\left(W^{0}(\zeta, s)\right)^{2} d \zeta=O\left(e^{-s / 2} \sqrt{s}\right) \rightarrow 0$ as $s \rightarrow \infty$. We conclude that, for large $s$, the solution $W^{0}(\zeta, s)$ of (5.4) is also an approximate solution of (5.3). Moreover, the nonlocal forcing term that makes the difference between (5.3) and (5.4) is uniform in the interval $[0,1]$.

Accordingly, we propose the ansatz

$$
u(x, t)=v(x, t)-p(t)+q(x, t)
$$

where the function $v(x, t)$ is a suitable solution of the equation with only local nonlinear forcing

$$
v_{t}=v_{x x}+v^{2}
$$

which blows up at the point $x=0$ at the time $t=T$. We anticipate that $p(t)$ will capture all of the spatially uniform nonlocal aspects of the blowup of $u$, so that $p(t)$ satisfies the differential equation

$$
\frac{d p}{d t}+p^{2}=\int_{0}^{1}(v-p+q)^{2} d x .
$$

We also anticipate that, while $v$ and $p$ both become very large (in different regions) as $t \rightarrow T$, the "remainder" $q$ will stay comparatively small. In particular, the contribution of the integral $q$ in the equation

$$
\int_{0}^{1}(p-v) d x=\int_{0}^{1} q d x
$$

(which is simply a variant of the integral constraint (1.4)) can be neglected, so that an estimate of $p$ can be made from this integral relation alone. This is slightly simpler than solving (5.8), and, provided that the contribution of $q$ can indeed be shown to be negligible, it is, in fact, equivalent. The development of the asymptotic investigation of $v, p$, and $q$ is therefore concluded with an examination of $q$, which serves to confirm a self-consistent overall picture.

The local asymptotic behaviour of $v(x, t)$ near the point of blowup is already well understood [3], [8], [13]. Its structure falls into distinct, but matching, asymptotic forms depending upon whether $x^{2} \leqq O[(T-t) s],(T-t) s \leqq O\left(x^{2}\right)$, or $x \neq O(1)$ as $t \rightarrow T$ 
and/or $x \rightarrow 0$. Thus, following [8],

$$
\begin{aligned}
v^{-1}= & (T-t)\left[1-\frac{1}{4} \xi^{-1}+O\left(\frac{\log ^{2}(\xi)}{\xi^{2}}\right)\right] \\
& +\frac{x^{2}}{8 \xi}\left[1+\xi^{-1}\left(\frac{5}{4} \log (\xi)+\log \left(1+\frac{\zeta^{2}}{8 \xi}\right)\right)\right]
\end{aligned}
$$

as $\xi=\alpha+s \rightarrow \infty$ for $x^{2}=O[(T-t) s]$, and

$$
\begin{aligned}
v^{-1}= & (T-t)\left(1-\frac{1}{4} \lambda^{-1}\right) \\
& +\frac{x^{2}}{8 \lambda}\left[1+\lambda^{-1}\left(\frac{1}{4} \log (\lambda)+\log \left(1+\frac{8 \lambda}{\zeta^{2}}\right)\right)+O\left(\frac{\log ^{2}(\lambda)}{\lambda^{2}}\right)\right]
\end{aligned}
$$

as $\lambda=\alpha-\log \left(x^{2} / 8\right) \rightarrow \infty$ for $(T-t) s=O\left(x^{2}\right)$. The constant $\alpha$ is a free parameter of the asymptotic structure that is related to the initial and boundary conditions and, for our nonlocal problem, to the integral constraint.

Away from the point of blowup $x=0$, the function $v$ remains bounded; that is, for $x \neq 0$,

$$
v=\bar{v}(x)+O(T-t),
$$

where, in the limit as $x \rightarrow 0, \bar{v}(x)$ must be consistent with (5.11) with $T-t$ set to zero, but is otherwise arbitrary. In this, it may be noted that formulae (5.11) and (5.12) offer asymptotic descriptions of the behaviour of $v$ even at the blowup time $t=T$ when formula (5.10) is inapplicable at any $x \neq 0$. The suitability of these asymptotic solutions in describing both $v$ and $u$ was demonstrated by the numerical calculations presented in Fig. 1.2. We now examine the asymptotic behaviour of $p(t)$ and $q(x, t)$ as blowup proceeds.

Using $v^{(\mathrm{i})}$ to denote the "inner" asymptotic solution $(5.10)$ and $v^{(\mathrm{o})}$ to denote the "outer" solution (5.11) and noting that

$$
\lambda=\xi-\log \left(\zeta^{2} / 8\right),
$$

the difference between the two asymptotic solutions can be shown to satisfy

$$
\begin{aligned}
\frac{v^{(\mathrm{o})}-v^{(\mathrm{i})}}{v^{(\mathrm{i})}}= & \frac{\frac{1}{4}-\zeta^{2} / 8 \xi\left[\frac{5}{4}+\log \left(\zeta^{2} / 8\right)\right]}{1+\zeta^{2} / 8 \xi} \xi^{-2} \log \left(\zeta^{2} / 8\right) \\
& +\frac{O\left(\zeta^{2} \log ^{2}(\xi) / \xi^{3}\right)}{1+\zeta^{2} / 8 \xi} \\
\approx & -\xi^{-2} \log ^{2}\left(\zeta^{2} / 8\right)
\end{aligned}
$$

the latter limit applying when $\zeta^{2} \gg \xi$. With this, we can estimate the integral

$$
\begin{aligned}
\int_{0}^{1} v d x= & \int_{0}^{1} v^{(\mathrm{i})} d x+O\left(\frac{1}{\sqrt{T-t}} \int_{\sqrt{\xi}}^{\infty} \frac{\xi^{-2} \log ^{2}\left(\zeta^{2} / 8\right)}{1+\zeta^{2} / 8 \xi} d \zeta\right) \\
= & \sqrt{8 \xi /(T-t)} \int_{0}^{\infty}\left[1+\frac{\xi^{-1}}{1-\nu^{2}}\left(\frac{1}{4}-\frac{5}{4} \nu^{2} \log (\xi)\right.\right. \\
& \left.\left.-\nu^{2} \log \left(1+\nu^{2}\right)\right)\right] \frac{d \nu}{1+\nu^{2}} \\
& +O\left(\frac{\xi^{-3 / 2} \log ^{2}(\xi)}{\sqrt{T-t}}\right) .
\end{aligned}
$$


Evaluating these integrals and neglecting the contribution of $q$ in (5.9), we obtain the following asymptotic estimate for $p$ :

$$
\begin{gathered}
p(t)=\sqrt{2 \xi /(T-t)} \pi\left[1-\xi^{-1}\left(\frac{5}{8} \log (\xi)+\frac{3}{8}+\log (2)\right)\right. \\
\left.+O\left(\xi^{-2} \log ^{2}(\xi)\right)\right]
\end{gathered}
$$

at large values of $\xi$.

To justify this asymptotic estimate, we must now consider the remainder term $q$, which satisfies the equation

$$
q_{t}=q_{x x}-2 p v+2(v-p) q+q^{2} .
$$

In estimating the behaviour of $q$, we can make use of leading-order estimates of the "forcing" terms $p v$ and $v-p$, which, from (5.10), (5.11), and (5.16), become

$$
p v \approx \begin{cases}\sqrt{2 \xi /(T-t)} \pi\left(T-t+x^{2} / 8 \xi\right)^{-1} & \text { for } x^{2}=O[(T-t) \xi], \\ \sqrt{2 \xi /(T-t)} \pi \bar{v}(x) & \text { for } x^{2} \gg(T-t) \xi\end{cases}
$$

and

$$
v-p \approx \begin{cases}\frac{1}{T-t+x^{2} / 8 \xi}-\sqrt{2 \xi /(T-t)} \pi & \text { for } x^{2}=O[(T-t) \xi] \\ \bar{v}(x)-\sqrt{2 \xi /(T-t)} \pi & \text { for } x^{2} \gg(T-t) \xi\end{cases}
$$

where

$$
\bar{v}(x) \approx \frac{8 \log \left(8 / x^{2}\right)}{x^{2}}
$$

to leading order as $x \rightarrow 0$.

For values of $x$ not close to zero, the following leading-order asymptotic behaviour for $q$ can be deduced:

$$
q \approx \bar{q}(x)+\sqrt{2 \xi(T-t)} 4 \pi(\bar{v}(x)+\bar{q}(x)),
$$

where $\bar{q}(x)$ is the value of $q$ at $t=T$ for $x \neq 0$. To justify (5.16), we must consider the behaviour of $q$ in the range $x^{2}=O[(T-t) \xi]$. In this range, $v \gg p$, and so $p$ can be neglected in comparison to $v$. Also, anticipating (as will be shown) that $q=$ $O(\sqrt{\xi /(T-t)})$ in the region of positive blowup, it can be seen that the nonlinear term of (5.17) should also be negligible to leading order. Defining

$$
q=g / \sqrt{T-t}
$$

(5.17) becomes

$$
g_{\xi}+\frac{1}{2} g+\frac{\zeta}{2} g_{\zeta}=g_{\zeta \zeta}+\frac{2}{1+\zeta^{2} / 8 \xi}(g-\pi \sqrt{2 \xi})+\text { h.o.t. }
$$

(where h.o.t. means higher-order terms), which has the leading-order asymptotic solution

$$
g \approx \frac{4}{3} \pi \sqrt{2 \xi} \frac{1+3 \zeta^{2} / 8 \xi}{\left(1+\zeta^{2} / 8 \xi\right)^{2}}
$$


It may be noted that any component of $g$ that is a solution of the homogeneous part of (5.23) can (and should) be absorbed into the leading-order blowup function $v$. Thus, only the inhomogeneous part of $g$ is retained in (5.24), and the function $v$ is rightly used to represent all of the local aspects of the positive blowup of $u$ that are consistent with (5.7).

We can now evaluate the integral of $q$ (to leading order). In the same way that the inner solution $v^{(i)}$ determines the integral of $v$ in (5.15), we have

$$
\int_{0}^{1} q d x \approx \frac{16}{3} \pi \xi \int_{0}^{\infty} \frac{1+3 \nu^{2}}{\left(1+\nu^{2}\right)^{2}} d \nu=\frac{16}{3} \pi^{2} \xi
$$

This serves to confirm that the contribution of $q$ in the integral (5.9) can be neglected in comparison to $p$ and $v$, justifying the asymptotic estimate for $p$.

By using (5.13), we can also use result (5.24) to deduce the leading-order asymptotic behaviour of $q$ for values of $x$ close, but not equal, to zero at times $t$ in the range $(T-t) \xi=O\left(x^{2}\right)$. That is,

$$
q \approx \frac{4}{3} \pi \sqrt{2 \lambda} \frac{T-t+3 x^{2} / 8 \lambda}{\left(T-t+x^{2} / 8 \lambda\right)^{2}} \sqrt{T-t} .
$$

This result shows that $q$ actually tends to zero as $t \rightarrow T$ for $x \neq 0$. Thus, for (5.20) to be consistent with this result, $\bar{q}(x)$ must be such that

$$
\lim _{x \rightarrow 0} \bar{q}(x)=0
$$

so that the local blowup of $q$ in the range $x^{2}=O[(T-t) \xi]$, given by (5.21) and (5.23), is more closely connected with the time-dependent term than with the leading-order term of (5.21).

If we now combine formulae (5.6), (5.11), and (5.16) with our estimates for $q$, we may deduce that, if $x$ is not small, then

$$
\begin{aligned}
& u(x, t) \sim-\frac{\sqrt{2} \pi}{(T-t)^{1 / 2}}|\log (T-t)|^{1 / 2} \\
& \cdot\left[1-\frac{5}{8} \frac{\log |\log (T-t)|}{|\log (T-t)|}+O\left(\frac{1}{s}+e^{-s / 2}\right)\right],
\end{aligned}
$$

where the unknown constant $\alpha$ is now included as part of the error term.

Moreover, we may also estimate the location of the zero $x^{*}$ of $u$ by determining the value $x^{*}$ for which $p(t)=v\left(x^{*}, t\right)+q\left(x^{*}, t\right)$. A simple but tedious calculation then shows that $x^{*}$ satisfies the following asymptotic relation:

$$
\left(x^{*}\right)^{2} \sim \frac{2 \sqrt{2}}{\pi}(T-t)^{1 / 2} s^{1 / 2}\left(1-\frac{7}{8} \frac{\log s}{s}+O\left(\frac{1}{s}\right)\right) .
$$

So, to leading order, we have

$$
u(1, t) \sim-\frac{\sqrt{2} \pi}{(T-t)^{1 / 2}}|\log (T-t)|^{1 / 2}, \quad\left(x^{*}\right)^{2} \sim \frac{2 \sqrt{2}}{\pi}(T-t)^{1 / 2}|\log (T-t)|^{1 / 2} .
$$

In these calculations, we have not determined the value of $\alpha$, which depends upon the initial conditions.

6. Numerical calculations for blowing up solutions. To verify the asymptotic formulae obtained in the previous section and, in particular, to test formulae (5.28), 
(5.29), we have made some careful numerical calculations of the solutions of (1.2), (1.4) for a variety of initial data. In particular, we have studied the following examples of initial data:

(i) $u_{0}(x)=30 \cos (\pi x)$,

(ii) $u_{0}(x)=100 \cos (\pi x)$,

(iii) $u_{0}(x)=100(\cos (\pi x)+\cos (2 \pi x))$.

This data is chosen so that the solution $u(x, t)$ blows up most rapidly at $x=0$.

For each of cases (i), (ii), and (iii), we record the value of the blowup time $T$ and the evolution of $u(1, t)$ and the zero location $x^{*}(t)$.

Formulae (5.28), (5.29) imply that close to the blowup time the following asymptotic identities should relate $u(1, t)$ and $x^{*}(t)$ to $\theta \equiv \log (u(0, t))$ :

$$
a(\theta) \equiv-e^{-\theta / 2} \frac{u(1, t)}{\sqrt{\theta}} \sim \sqrt{2} \pi\left(1-\frac{5}{8} \frac{\log (\theta)}{\theta}+O\left(\frac{1}{\theta}\right)\right)
$$

and

$$
b(\theta) \equiv \frac{e^{\theta / 2}}{\sqrt{\theta}}\left(x^{*}\right)^{2} \sim \frac{2 \sqrt{2}}{\pi}\left(1-\frac{7}{8} \frac{\log (\theta)}{\theta}+O\left(\frac{1}{\theta}\right)\right) .
$$

Accordingly, in Figs. 6.1 and 6.2, we present numerically computed graphs of $a(\theta)$ and $b(\theta)$ as functions of $1 / \theta$ for $0.035<1 / \theta$. In these figures, the last data point is marked with a circle and the graphs are linearly extrapolated back to the line $1 / \theta=0$.

It is very clear from these figures that there is close agreement between the numerical calculations and the leading-order form of the asymptotic formulae (6.1), (6.2). In particular, the constants $\sqrt{2} \pi$ and $2 \sqrt{2} / \pi$ are accurately predicted by the

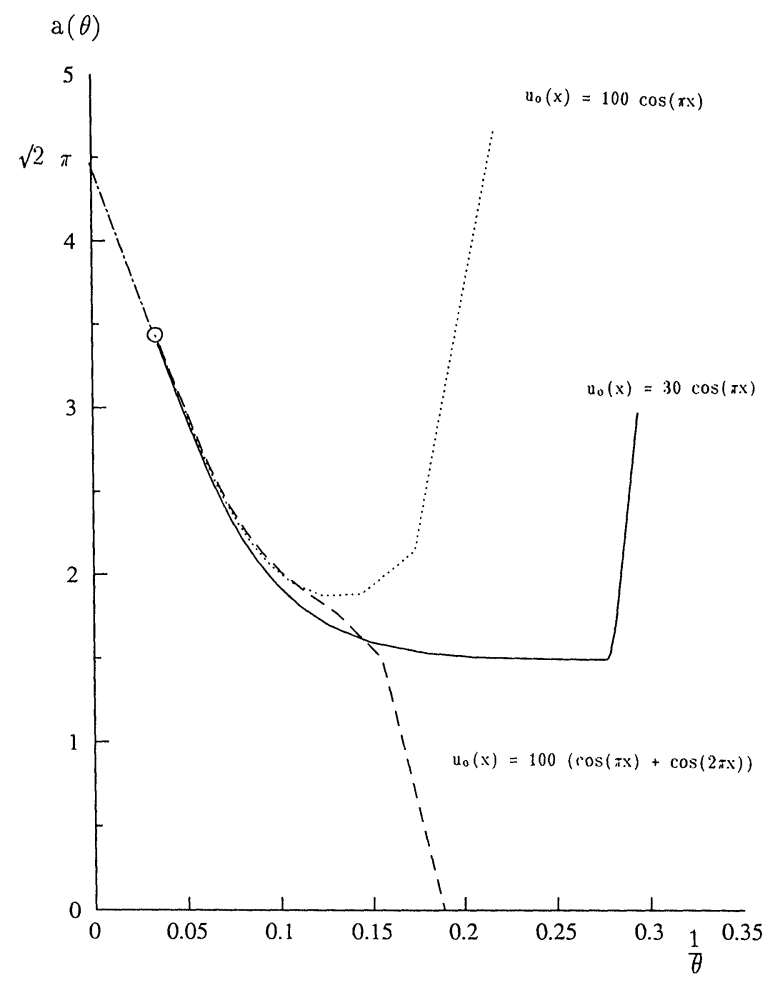

FIG. 6.1. Graph of $a(\theta)$ given by (6.1), as a function of $1 / \theta$ for various forms of the initial data. 


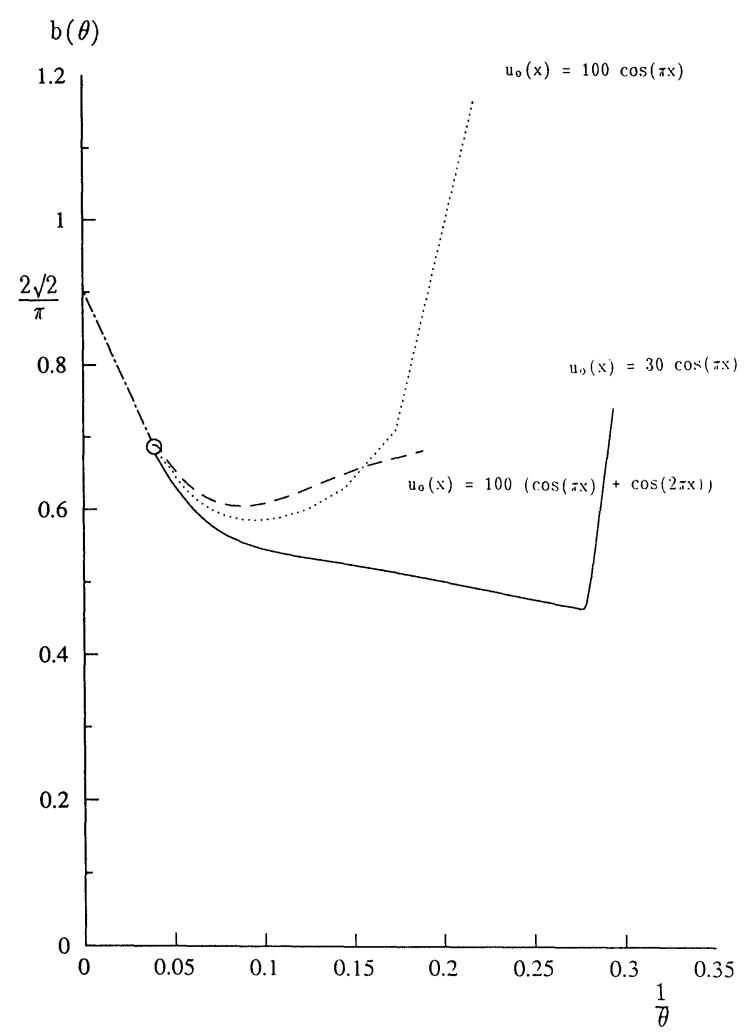

FIG. 6.2. Graph of $b(\theta)$ given by (6.2), as a function of $1 / \theta$ for various forms of the initial data.

numerics. It is also clear from the figures that, for the range of values considered, the dominant error from the leading order takes the form $\gamma / \theta$. Here $\gamma$ appears to be independent of the initial conditions. The terms of the form $\gamma / \theta$ dominate the terms of the form $(\log (\theta)) / \theta$ for all but very large values of $\theta^{-1}$.

The figures demonstrate that the asymptotic formulae are most accurate when $1 / \theta<0.05$, that is, when $u(0, t)>4.8 \times 10^{8}$. For these values, the width of the main peak is of order $\sqrt{u(0, t)}<5 \times 10^{-5}$, which gives an upper bound to the size of the mesh used by the numerical computation in the neighbourhood of $x=0$. Thus, to compute the solutions accurately, we seek to place a reasonable number of mesh points within the main peak of the function $u(x, t)$ while still using an efficient computational scheme. Straightforward rescaling algorithms, which attempt to shift the points in a spatial grid progressively into the region of positive blowup [8], [14] are not appropriate because an adequate resolution of regions where $u$ grows more slowly (and negatively) must nevertheless also be maintained.

To maintain a "good" spatial resolution of all aspects of the growth of $u$, two approaches were developed. These approaches rely on the definition of a weighting function $W(x)$ defined, numerically, at a set of grid points $x=x(i), i=0, \ldots, N$, with $x(0)=0$ and $x(N)=1$. For a point distribution that is weighted according to a given function $W(x)$, the points $x(i)$ are distributed such that

$$
\int_{i}^{i+1} W(x(i)) d i=\frac{1}{N} \int_{0}^{N} W(x(i)) d i
$$

for each $i=0, \ldots, N-1$, assuming a suitable interpolation. In arriving at the two 
approaches used in maintaining a suitable resolution, we denote the numerically stored values of $u(x(i), t)$ at any time $t$ by $u(i)$. defining

Assuming that the maximum value of $u$ is $u_{m}$, our first approach is obtained by

$$
W^{2}(x(i))=\left[\left(\frac{d x}{d i}\right)^{2}+\left(\frac{d u / d i}{u_{m}}\right)^{2}\right]+M^{2}\left[\left(\frac{d^{2} x}{d i^{2}}\right)^{2}+\left(\frac{d^{2} u / d i^{2}}{u_{m}}\right)^{2}\right]
$$

for a suitably large value of $M$. In our calculations, we found that taking $M=5$ to $M=8$ worked well in conjunction with high-order differencing methods for calculating derivatives and interpolating the weighting function $W$. Formula (6.3) represents a weighting function that distributes the positions of the grid points to resolve both arc length and curvature. If $M=0$, then points are distributed with respect to arc length on a graph of $u / u_{m}$ versus $x$, and, if $M \rightarrow \infty$, then points are equidistributed with respect to curvature.

Assuming that there is a single point of positive blowup at $x=x_{m}$, where $u=u_{m}$, a second approach relies upon the assumption that the positively growing peak of $u$ varies locally in approximate proportion to $\left(1+\left(\left(x-x_{m}\right) / x_{d}\right)^{2}\right)^{-1}$, where $x_{d}(t)$ represents the "width" of the peak at any time. It can be seen that this structure is consistent with the asymptotic structure for the blowup examined in the previous section. A suitable weighting function that takes advantage of this is obtained by defining

$$
W(x)=1+M /\left[x_{d}+\frac{\sin ^{2}\left(\pi\left(x-x_{m}\right) / 2\right)}{x_{d} \pi^{2} / 4}\right]
$$

with $x_{d}(t)$ estimated from the solution at any time such that $u\left(x_{m}+x_{d}\right)=u_{m} / 4$. The value of $M$ is chosen to increase or decrease the proportion of points within the region of the peak, with a value of $M=1$ proving quite adequate. The sine function used in this definition for $W(x)$ recognises the periodicity of 2 implied by the Neumann boundary conditions for $u$.

The accuracy of time integration was improved by using a directly calculated Taylor-series approach, truncated at the sixth time derivative. That is, by differentiating (1.2) and (1.6) five times with respect to time, the resulting formulae were used to provide numerical estimates for the time derivatives up to $\partial^{6} / \partial t^{6} u(x, t)$ at each timestep. Timestepping was then performed using a correspondingly truncated Taylor series to maintain a high accuracy, with timesteps progressively reduced as the blowup proceeds.

Both methods of point redistribution were found to work well in following the progress of blowup to maximum values of $u$ of the order of $10^{12}$ using as few as 100 points. About half of these were typically used to resolve the structure of the peak while the others ensured that the remainder of the evolution of $u$ was adequately resolved. Differences between the two approaches are indiscernible in Figs. 6.1 and 6.2.

Appendix. Let $p>1$ be a positive integer. We consider the problem

$$
u_{t}=u_{x x}+u^{p}-\int_{0}^{1} u^{p} d x
$$

with the boundary conditions

$$
u_{x}(0, t)=u_{x}(1, t)=0
$$

and initial data satisfying

$$
\int_{0}^{1} u(x, 0) d x=0
$$


Note that $\int_{0}^{1} u(x, t) d x=0$ for all subsequent times. We apply energy arguments (see, for example, [1]) to establish blowup results for this problem. If $p$ is odd, we may prove blowup in a finite time. If $p$ is even, we establish blowup in, at least, infinite time. The results for even $p$ are not sharp since we established in $\S 4$ that blowup may occur if $p=2$. Throughout this appendix, $\|\cdot\|$ denotes the $L_{2}$ norm in the spatial variable $x$.

Theorem A1. Consider (A1)-(A3). Define

$$
E=\frac{2}{p+1} \int_{0}^{1} u(x, 0)^{p+1} d x-\left\|u_{x}(x, 0)\right\|^{2}
$$

and consider initial data satisfying (A3) and $E>0$. (i) If $p$ is even, then

$$
\|u(x, t)\|^{2} \geqq\|u(x, 0)\|^{2} e^{(p-1) \pi^{2} t}+\frac{(p+1) E}{(p-1) \pi^{2}}\left[e^{(p-1) \pi^{2} t}-1\right] .
$$

(ii) If $p$ is odd, then $\|u(x, t)\| \rightarrow \infty$ as $t \rightarrow T<\infty$.

Proof. Multiplying (A1) by $u$ and integrating over $0<x<1$, we obtain, upon integration by parts,

$$
\frac{1}{2} \frac{d}{d t}\|u\|^{2}=-\left\|u_{x}\right\|^{2}+\int_{0}^{1} u^{p+1} d x .
$$

Multiplying (A1) by $u_{t}$ and integrating over $0<x<1$, we obtain

$$
\left\|u_{t}\right\|^{2}=-\frac{1}{2} \frac{d}{d t}\left(\left\|u_{x}\right\|^{2}\right)+\frac{1}{p+1} \frac{d}{d t} \int_{0}^{1} u^{p+1} d x
$$

Integrating gives

$$
-\left\|u_{x}\right\|^{2} \geqq E-\frac{2}{p+1} \int_{0}^{1} u^{p+1} d x .
$$

We now observe that $E$ can be made positive by choosing suitably large initial data. Using (A4) and (A5), we now prove parts (i) and (ii) separately.

For $p$ even, we eliminate the integral of indefinite sign and obtain

$$
\frac{d}{d t}\|u\|^{2} \geqq(p-1)\left\|u_{x}\right\|^{2}+(p+1) E .
$$

Using the Poincare inequality for arbitrary functions in the space $H^{1}(0,1)$ with $\int_{0}^{1} u d x=0[4]$, we obtain

$$
\frac{d}{d t}\|u\|^{2} \geqq(p-1) \pi^{2}\|u\|^{2}+(p+1) E .
$$

Integrating this differential inequality yields result (i).

For $p$ odd, we eliminate $\left\|u_{x}\right\|^{2}$ and obtain

$$
\frac{1}{2} \frac{d}{d t}\|u\|^{2} \geqq \frac{(p-1)}{(p+1)} \int_{0}^{1} u^{p+1} d x+E .
$$

Since $p$ is odd, we may apply Hölder's inequality to obtain

$$
\frac{d}{d t}\|u\|^{2} \geqq 2 \frac{(p-1)}{(p+1)}\|u\|^{p+1}+2 E .
$$


By standard comparison arguments the solutions of this inequality blow up in a finite time since $p>1$.

Acknowledgments. The authors thank Professors J. T. Stuart and V. Galaktionov and Drs. M. Floater, A. Lacey, and Y. Tourigny for many helpful conversations.

\section{REFERENCES}

[1] J. M. BALL, Remarks on blow up and nonexistence theorems for nonlinear evolutionary equations, Quart. J. Math. Oxford Ser., 28 (1977), pp. 473-486.

[2] J. Bebernes, A. Bressan, AND A. A. LACEy, Total blow-up versus single point blow-up, J. Differential Equations, 73 (1988), pp. 30-44.

[3] M. BERGER AND R. V. KOHN, A rescaling algorithm for the numerical calculation of blowing up solutions, Comm. Pure Appl. Math., 41 (1988), pp. 841-863.

[4] H. BreZIS, Analyse fonctionnalle, Masson, Paris, 1983.

[5] N. CHAFEE, Asymptotic behavior for solutions of a one-dimensional parabolic equation with homogeneous Neumann boundary conditions, J. Differential Equations, 18 (1975), pp. 111-135.

[6] S. Childress, Chemotactic collapse in two dimensions, in Modelling of Patterns in Space and Time, Lecture Notes in Biomathematics 55, Springer-Verlag, Berlin, New York, 1984.

[7] S. Childress And J. K. Percuss, Nonlinear aspects of chemotaxis, Math. Biosci., 56 (1981), pp. 883-901.

[8] J. W. Dold, On asymptotic forms of reactive-diffusive runaway, Proc. Roy. Soc. London Ser. A, 433 (1991), pp. 521-545.

[9] S. DoOLE, Constructive aspects of steady state and dynamic bifurcation, M.Sc. thesis, University of Bath, UK, 1990.

[10] C. M. ELLIOTt, The Cahn-Hilliard model for the kinetics of phase separation, in Mathematical Models for Phase Change Problems, J. Rodrigues, ed., Birkhäuser-Verlag, Basel, Boston, 1989.

[11] A. Friedman ANd B. MCLeOd, Blow up of positive solutions of semilinear heat equations, Indiana Univ. Math. J., 34 (1985), pp. 425-447.

[12] J. FURTER AND M. GRINFELD, Local vs. non-local interactions in population dynamics, J. Math. Biol., 27 (1989), pp. 65-80.

[13] V. Galaktionov, On blow-up and degeneracy for the semi-linear heat equation with source, Proc. Roy. Soc. Edinburgh Ser. A, 115 (1990), pp. 19-24.

[14] Y. GigA AND R. V. KoHn, Asymptotically self similar blowup of semilinear heat equations, Comm. Pure Appl. Math., 38 (1985), pp. 297-319.

[15] D. Henry, Geometric Theory of Semilinear Parabolic Problems, Springer Lecture Notes in Mathematics, $840,1978$.

[16] A. A. LACEY, Global blow-up of a nonlinear heat equation, Proc. Roy. Soc. Edinburgh Ser. A, 104 (1986), pp. 161-167.

[17] H. A. LEVINE, The role of critical exponents in blowup theorems, SIAM Rev., 32 (1990), pp. 262-288.

[18] R. PAlAIS, Blowup for nonlinear equations using a comparison principle in Fourier space, Comm. Pure Appl. Math., 41 (1988), pp. 165-196.

[19] J. RuBENSTEIN AND P. STERNBERG, Non-local reaction diffusion equations and nucleation, submitted.

[20] R. SCHAAF, Stationary solutions of chemotaxis systems, Trans. Amer. Math. Soc., 292 (1985), pp. 531-556.

[21] J. SMOller AND A. WASSERMAN, Global bifurcation of steady state solutions, J. Differential Equations, 39 (1981), pp. 269-290.

[22] J. W. DolD, A note on integral properties of periodic orbits, SIAM Rev., 35 (1993), pp. 125-129. 